\title{
RADICAIS LIVRES DE OXIGÊNIO: UM SOFTWARE INTRODUTÓRIO
}

\author{
Daniela K. Yokaichiya e Eduardo Galembeck
}

Departamento de Bioquímica - IB - Unicamp - CP 6109 - 13083-970 - Campinas - SP

Bayardo B. Torres

Departamento de Bioquímica - Instituto de Química, USP - CP 26077 - 05508-900 - São Paulo - SP

Recebido em 9/11/98; aceito em 7/5/99

\begin{abstract}
OXYGEN FREE RADICALS: AN INTRODUCTORY SOFTWARE. Though Free Radicals is one of the most frequently explored scientific subjects in mass communication media, the topic is absent of many Biochemistry introductory courses, especially those in which the students do not have a good chemical background. To overcome this contradictory situation we have developed a software treating this topic in a very simple way. The software is divided in four sections: (1) definition and description of free radicals, (2) production pathways, (3) mechanism of action and (4) enzymatic and non enzymatic protection. The instructional capacity of the software has been both qualitative and quantitatively evaluated through its application in undergraduate courses. The software is available in the INTERNET at the site: http://www.unicamp.br/ib/bioquimica/ensino.
\end{abstract}

Keywords: free radicals; educational software; C.A.I.

\section{INTRODUÇÃO}

Radicais Livres é um dos temas de pesquisa mais intensamente investigado nos últimos anos, tanto em seus aspectos básicos quanto nas aplicações clínicas. Pela sua abrangência e pela possibilidade que o seu estudo gerou na prevenção e cura de diversas moléstias, o tema tem recebido ampla divulgação pelos meios de comunicação, tendo se tornado, rapidamente, de domínio público. O tratamento dado ao assunto pela mídia é, muitas vezes, revestido de algum sensacionalismo e, quase sempre, destituído do rigor e da prudência desejáveis na apresentação de conclusões que ainda não são consensuais entre os especialistas. Em contraposição à freqüência com que o assunto é noticiado pela imprensa leiga, este não é um tópico rotineiramente abordado nas disciplinas de Bioquímica para a graduação. Uma das prováveis razões dessa ausência é a falta de uma literatura adequada, destinada especialmente a alunos que não possuem conhecimentos sólidos em Química. O tratamento dado ao tema na maioria dos livros-texto exige conhecimentos químicos que muitos alunos da área biológica não possuem. Em casos como este, a Instrução Assistida por Computador (CAI) apresenta-se como uma estratégia atraente, pela possibilidade de apresentar um tema específico, que pode ser seguido por cada aluno segundo seu próprio ritmo e conhecimento prévio. Como recurso auxiliar ou introdutório ao tratamento do tópico Radicais Livres, desenvolvemos um software que trata o assunto de forma condensada mas simples, permitindo sua utilização em qualquer curso de graduação.

\section{DESCRIÇÃO DO SOFTWARE}

O programa é constituído de quatro seções, com subdivisões (Figura 1): (A) Fontes de produção - Descreve as fontes de produção mais conhecidas e enfatiza o papel da cadeia de transporte de elétrons na formação dos radicais livres de oxigênio (Figura 2); (B) $O$ que são e como se formam - Demonstra a estrutura química dos radicais livres e ilustra a formação dos diferentes radicais livres de oxigênio na cadeia de transporte de elétrons (Figura 3); (C) Como agem e o que causam - Descreve os mecanismos de ação e os efeitos provocados (Figura 4); (D) Mecanismos de proteção - Apresenta os mecanismos enzimáticos (glutationa peroxidase, superóxido dismutase e catalase) e

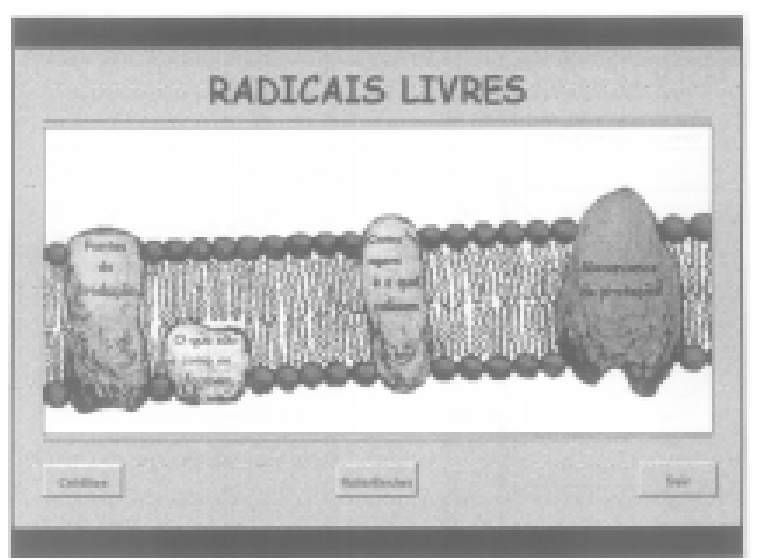

Figura 1. Tela de abertura do software. O clique do mouse sobre cada complexo da cadeia de transporte de elétrons abre a seção correspondente ao título.

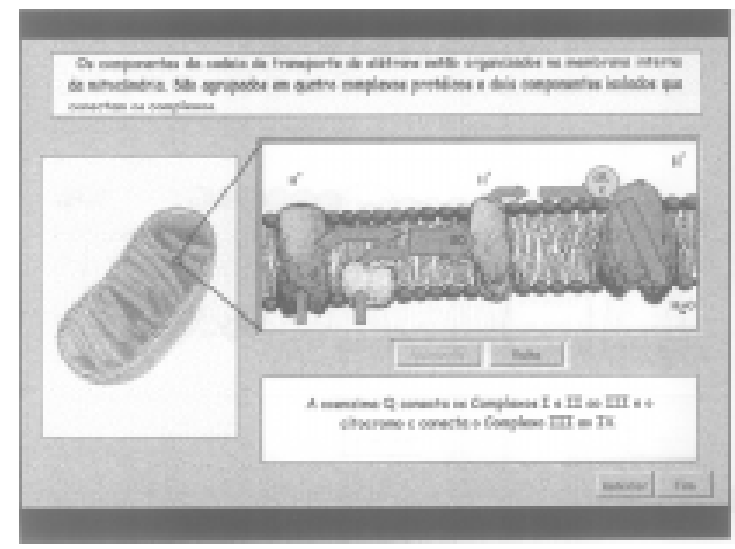

Figura 2. Esquema das tranferências de elétrons processadas na cadeia de transporte de elétrons mitocondrial, uma das principais fontes de produção de radicais livres de oxigênio. 


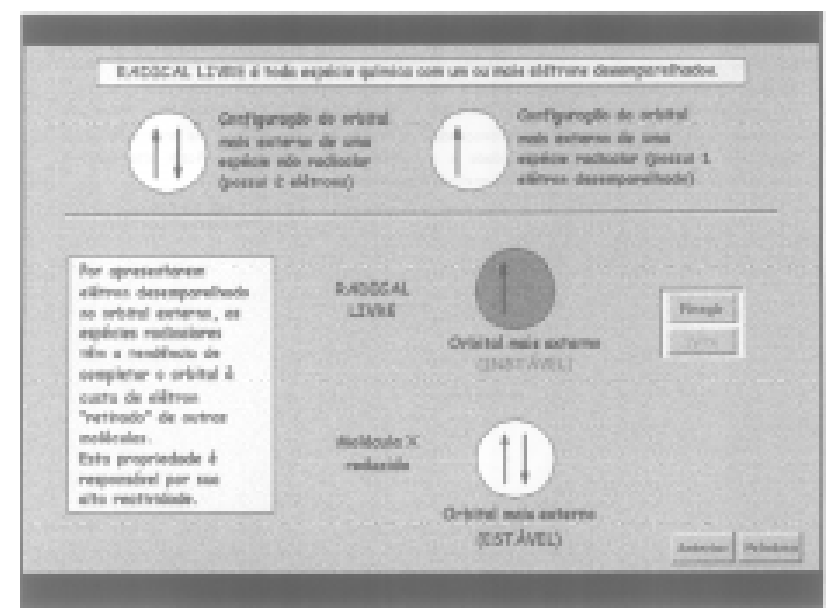

Figura 3. Tela explicativa da reatividade dos radicais livres, abrindo uma animação sobre a sua formação seqüencial.

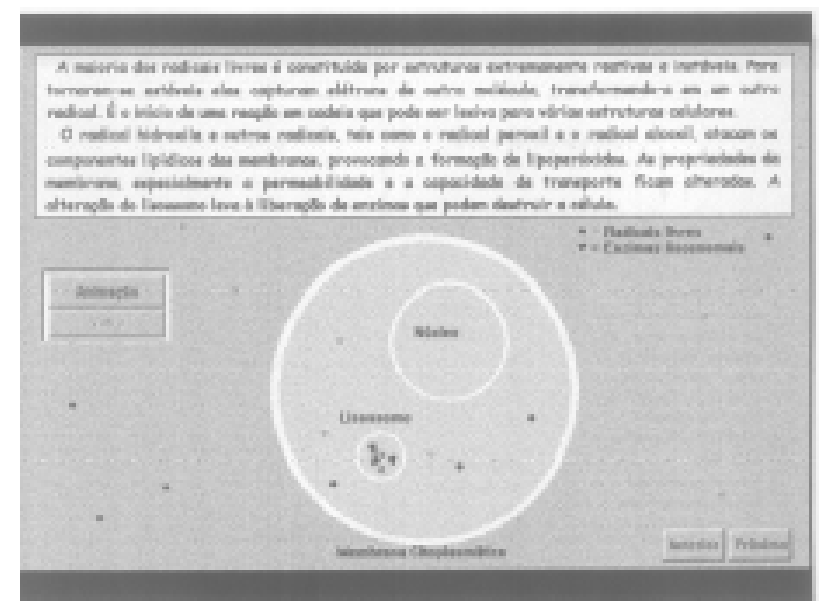

Figura 4. Animação ilustrando os processos que levam a lesões de membranas celulares provocadas pelos radicais livres.

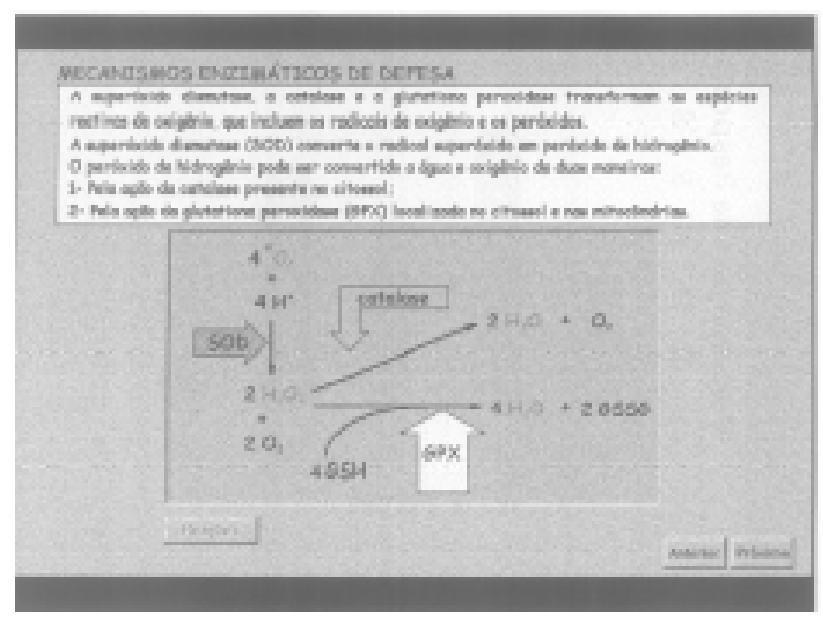

Figura 5. Esquema da conversão enzimática de radicais livres a compostos menos reativos. A figura mostrada na tela é o resultado final de uma seqüência de reações mostradas em animação.

não-enzimáticos (vitaminas $\mathrm{C}$ e $\mathrm{E}$ e glutationa) de proteção (Figura 5). Os conteúdos de todas as seções são simplificados a partir de textos consagrados e acessíveis à consulta ${ }^{1,2,3,4}$.

As seções são desenvolvidas através de imagens associadas a pequenos textos. Quando pertinente, estão incluídas animações, para auxiliar a compreensão dos processos essencialmente dinâmicos. Questões de múltipla escolha, que permitem o acompanhamento do próprio aprendizado, estão inseridas em etapas críticas; o acerto nas respostas não é limitante para o prosseguimento no programa, pois as questões são respondidas em telas seguintes.

O software foi desenvolvido utilizando-se um autor de multimídia, o ToolBook II Instructor ${ }^{5}$ e é compatível com PC 486 ou superior, que rode o sistema operacional Windows 95/ 98 e NT.

\section{AVALIAÇÃo}

O software foi produzido para atender a uma situação específica de ensino: a disciplina de graduação Bioquímica da Nutrição, oferecida como eletiva pelo Departamento de Bioquímica da Unicamp, com corpo discente heterogêneo (alunos dos cursos de Biologia, Educação Física, Engenharia de Alimentos, Enfermagem, Medicina e Química). Um levantamento inicial permitiu verificar o nível e a origem das informações prévias sobre Radicais Livres dos alunos matriculados na disciplina. Através de um questionário, indagou-se se tais informações eram: (A) Insignificantes, (B) Decorrentes de informações veiculadas pela mídia (revistas, jornais, rádio, TV), (C) Provenientes de revistas de divulgação científica, (D) Resultantes de disciplinas cursadas na Universidade, ou (E) Originadas de outras fontes de informação.

A avaliação quantitativa do software foi procedida através de um questionário respondido pelos usuários do programa. $\mathrm{O}$ questionário era composto de oito afirmações (Legenda da Figura 7) e os sujeitos escolhiam, para cada uma delas, a alternativa que melhor descrevesse sua opinião: Concordo Fortemente (CF), Concordo (C), Indeciso (I), Discordo (D) ou Discordo Fortemente (DF).

A avaliação qualitativa foi feita pelos professores e monitores da disciplina através da observação dos usuários durante a utilização do software e coleta de suas opiniões e considerações. A capacidade instrucional do programa pôde ser acompanhada pelos monitores porque, juntamente com o programa, foi distribuído aos usuários um roteiro contendo questões que deveriam ser respondidas apenas com as informações contidas no software.

\section{RESULTADOS E DISCUSSÃO}

Tratando-se de uma clientela heterogênea, a diversidade sobre o nível e a origem das informações prévias sobre o tema do software (Figura 6) era esperada. Grande parte dos estudan-

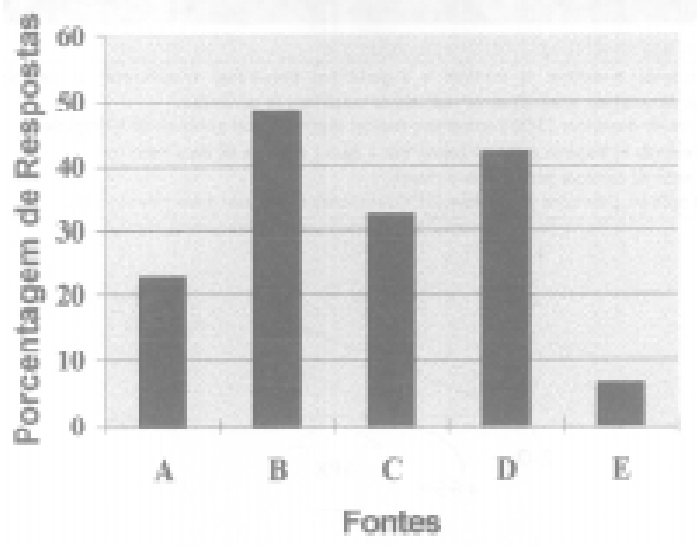

Figura 6. Distribuição de respostas sobre nível e fontes de informação sobre radicais livres dos alunos. (A) Insignificantes, (B) Informações pela mídia, (C) Informações por revistas de divulgação científica, $(D)$ Informação por disciplinas nas Universidades, (E) Outras Fontes. As opções não são excludentes, levando à soma maior do que $100 \%$. 


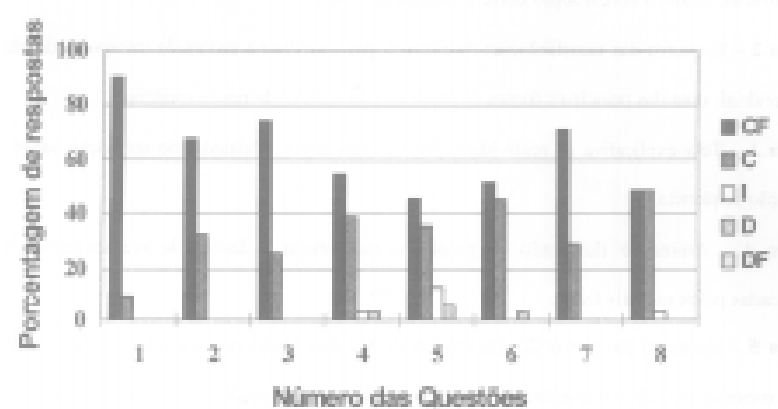

Figura 7. Distribuição das respostas às questões: (1) O software é de fácil manuseio, (2) A utilização deste tipo de recurso tem vantagens sobre textos, (3) O programa trouxe conhecimentos novos sobre Radicais Livres, (4) Os conceitos estão expostos de forma clara, (5) Meus conhecimentos anteriores foram suficientes para aprender com o software, (6) Os textos estão redigidos com clareza, (7) As animações auxiliam a compreender as reações químicas e os fenômenos exibidos, (8) Aprendi bastante sobre Radicais Livres com o programa. $C F=$ Concordo Fortemente, C=Concordo, $I=I n d e c i s o, D=D i s c o r d o$, DF $=$ Discordo Fortemente.

tes obteve informações através da mídia (48\%) e por revistas de divulgação científica (32\%). É notável, entretanto, que para apenas $42 \%$ dos alunos o aprendizado sobre Radicais Livres tenha se dado através de disciplinas da Universidade. Pode-se supor, portanto, que a maioria dos alunos de graduação tenha conceitos frágeis ou fragmentados sobre o assunto.

O software teve boa aceitação pelos alunos (Figura 7). De fato, a soma das respostas Concordo Fortemente e Concordo para todas as questões ultrapassa $90 \%$. Apesar da pretendida simplicidade no tratamento do tópico, os usuários foram menos enfáticos quanto à suficiência dos seus conhecimentos anteriores para o aprendizado proposto. Na avaliação qualitativa, recolhida pelos comentários dos alunos durante e após a utilização do programa, os pontos positivos ressaltados foram: a alta motivação resultante do dinamismo da aula, o respeito ao ritmo individual de aprendizagem, a concisão na abordagem do tema em comparação com os livros e o esclarecimento de mitos referentes ao tema. Por outro lado, foram apontadas as seguintes desvantagens: dificuldade na movimentação de uma seção para outra, linguagem contendo termos bioquímicos que dificultam a compreensão e necessidade de tempo longo para apreensão do conteúdo.

Embora o programa tenha sido planejado para utilização em laboratórios de informática, sua estrutura é compatível com a utilização como recurso auxiliar em aulas expositivas. O software está disponível no seguinte endereço: http://www.unicamp. br/ ib/bioquimica/ensino.

\section{AGRADECIMENTOS}

Os autores agradecem o Prof. Dr. Luís Eduardo Soares Netto pela revisão crítica do manuscrito e do software. Este trabalho foi financiado pelo projeto PROIN (CAPES) e pela FAPESP.

\section{REFERÊNCIAS}

1. Halliwell, B. \& Gutteridge, J. M. C.; Free Radical in Biology and Medicine; Claderon Press; Oxford, 1989.

2. Hollen, J. R.; Elements of General, Organic and Biological Chemistry; John Wiley \& Sons. Inc.; Nova York, 1995.

3. Lehninger, A. L.; Nelson, D. L. \& Cox, M. M.; Princípios de Bioquímica; Sarvier Editora; São Paulo,1995.

4. Meneghini, R.; Ciência Hoje 1987, 5, 28:57-62.

5. Toolbook II Instructor 6.1a. Copyright (C) 1990-1998. Asymetrix Learning Systems, Inc. 